\title{
Lymphatic drainage from bronchus-associated lymphoid tissue in tolerant lung allografts promotes peripheral tolerance
}

\author{
Wenjun Li, Jason M. Gauthier, ${ }^{1}$ Alice Y. Tong, ${ }^{1}$ Yuriko Terada, ${ }^{1}$ Ryuji Higashikubo, ${ }^{1}$ Christian C. Frye, ${ }^{1}$ Margaret S. Harrison, ${ }^{1}$ \\ Kohei Hashimoto, ${ }^{1}$ Amit I. Bery, ${ }^{2}$ Jon H. Ritter, ${ }^{3}$ Ruben G. Nava, ${ }^{1}$ Varun Puri, ${ }^{1}$ Brian W. Wong, ${ }^{1}$ Kory J. Lavine, ${ }^{2}$ Ankit Bharat, ${ }^{4}$ \\ Alexander S. Krupnick, ${ }^{5}$ Andrew E. Gelman, ${ }^{1,3}$ and Daniel Kreisel ${ }^{1,3}$
}

'Departments of Surgery, ${ }^{2}$ Medicine, and ${ }^{3}$ Pathology \& Immunology, Washington University in St. Louis, St. Louis, Missouri, USA. ${ }^{4}$ Department of Surgery, Northwestern University, Chicago, Illinois, USA. ${ }^{5}$ Department of Surgery, University of Maryland, Baltimore, Maryland, USA.

\begin{abstract}
Tertiary lymphoid organs are aggregates of immune and stromal cells including high endothelial venules and lymphatic vessels that resemble secondary lymphoid organs and can be induced at nonlymphoid sites during inflammation. The function of lymphatic vessels within tertiary lymphoid organs remains poorly understood. During lung transplant tolerance, Foxp $3^{+}$cells accumulate in tertiary lymphoid organs that are induced within the pulmonary grafts and are critical for the local downregulation of alloimmune responses. Here, we showed that tolerant lung allografts could induce and maintain tolerance of heterotopic donor-matched hearts through pathways that were dependent on the continued presence of the transplanted lung. Using lung retransplantation, we showed that Foxp $3^{+}$cells egressed from tolerant lung allografts via lymphatics and were recruited into donor-matched heart allografts. Indeed, survival of the heart allografts was dependent on lymphatic drainage from the tolerant lung allograft to the periphery. Thus, our work indicates that cellular trafficking from tertiary lymphoid organs regulates immune responses in the periphery. We propose that these findings have important implications for a variety of disease processes that are associated with the induction of tertiary lymphoid organs.
\end{abstract}

\section{Introduction}

Tertiary lymphoid organs can be induced in nonlymphoid tissues during states of chronic inflammation or antigen exposure and are structurally similar to secondary lymphoid organs. One such example is bronchus-associated lymphoid tissue, a pulmonary tertiary lymphoid organ that can be induced in murine and human lungs during a variety of inflammatory conditions, including respiratory infections, autoimmune diseases, chronic obstructive pulmonary disease, cancers, and pulmonary transplantation (1). Tertiary lymphoid organs are hallmarked by the development of high endothelial venules, which are specialized vascular structures that facilitate the entry of leukocytes into tertiary lymphoid organs (2). In addition, multiple studies have documented the existence of lymphatic vessels within tertiary lymphoid organs, including bronchus-associated lymphoid tissues, in both mice and humans $(3,4)$. Although the patterns and function of lymphatic flow in secondary lymphoid tissues have been the focus of many studies, relatively little is known about the role of lymphatic vessels in tertiary lymphoid organs. Afferent lymphatics transport soluble or cell-associated antigens to secondary lymphoid

Conflict of interest: DK and KJL have a pending patent titled "Compositions and methods for detecting CCR2 receptors" (application number 15/611,577).

Copyright: ( 2020 , American Society for Clinical Investigation.

Submitted: January 2, 2020; Accepted: September 3, 2020;

Published: November 16, 2020.

Reference information: J Clin Invest. 2020;130(12):6718-6727.

https://doi.org/10.1172/JCl136057. organs where their encounter with lymphocytes initiates adaptive immune responses. The importance of afferent lymphatics in tertiary lymphoid organs, however, remains to be established. It has been suggested that the continuous presence of antigen in tertiary lymphoid organs, such as autoantigen in autoimmune diseases or alloantigen in transplanted organs, obviates the need for antigen transport to these sites (5). Efferent lymphatics promote the egress of lymphocytes from secondary lymphoid organs and it has been reported that $\mathrm{CD} 4^{+} \mathrm{T}$ cells make up a large fraction of lymphocytes in efferent lymph (6). Several studies have documented the presence of lymphocytes within lymphatic vessels of tertiary lymphoid organs, suggesting that cellular trafficking occurs through these channels $(5,7)$.

We and others have observed that tertiary lymphoid organs are induced in transplanted tissues and organs, sites where alloantigen is continuously present for the life of the graft (8). In contrast to many studies that have suggested that tertiary lymphoid organs are deleterious and promote graft rejection, we have shown that Foxp $3^{+} \mathrm{T}$ lymphocytes are located within bronchus-associated lymphoid tissue that is induced in tolerant mouse lung allografts (9-11). Furthermore, we have demonstrated that selective depletion of Foxp $3^{+}$cells from tolerant grafts triggers antibody-mediated rejection, thereby establishing that Foxp $^{+}$cells suppress alloimmune responses locally (12). In this study, we employ a mouse lung retransplant model to show that $\mathrm{Foxp}^{+}$cells migrate from tolerant lung allografts to the periphery via lymphatic channels. The induction of peripheral tolerance depends on lymphatic drainage from the tolerant lung allograft. 
A

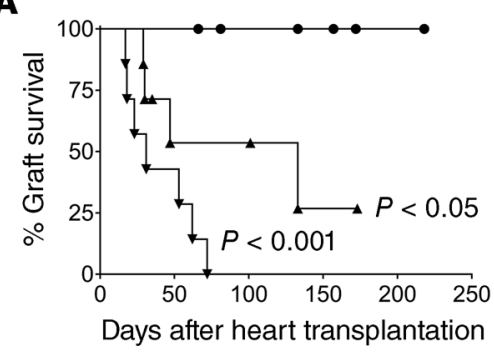

- $\mathrm{BALB} / \mathrm{C} \rightarrow \mathrm{B} 6$ (tolerant BALB/c lung)

* BALB/C $\rightarrow$ B6 (tolerant BALB/c lung + early PNX)

$\star \mathrm{BALB} / \mathrm{C} \rightarrow \mathrm{B} 6$ (tolerant BALB/c lung + late PNX)
B

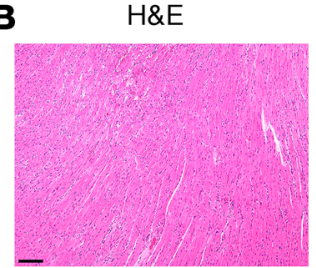

E

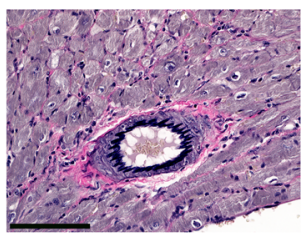

$\mathrm{BALB} / \mathrm{C} \rightarrow \mathrm{B} 6$ (tolerant BALB/c lung)

C

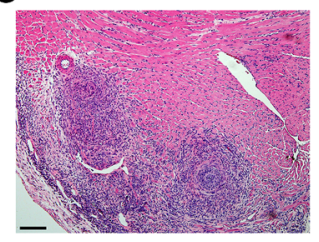

$\mathbf{F}$

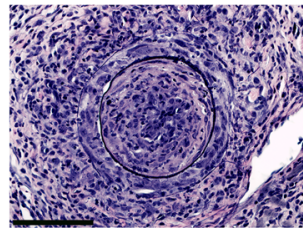

$\mathrm{BALB} / \mathrm{c} \rightarrow \mathrm{B} 6$ (tolerant BALB/c lung + early PNX)

\section{D}

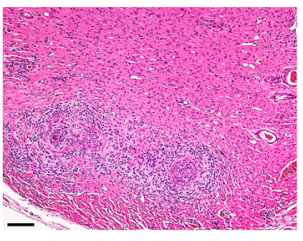

G

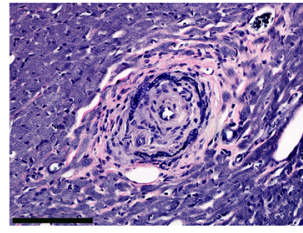

$\mathrm{BALB} / \mathrm{c} \rightarrow \mathrm{B} 6$ (tolerant $\mathrm{BALB} / \mathrm{c}$ lung + late PNX)

Figure 1. Induction and maintenance of peripheral tolerance after lung transplantation depend on the presence of the tolerant pulmonary allograft. (A) Kaplan-Meier survival curves of BALB/c hearts that were transplanted into B6 mice that had received BALB/c lungs under perioperative costimulatory blockade at least 30 days prior $(\bullet)$ without $(n=8),(\nabla)$ with removal of the tolerant pulmonary allograft 24 hours before cardiac transplantation (early pneumonectomy) $(n=7)$, and $(\boldsymbol{\Delta})$ with removal of the tolerant pulmonary allograft 30 days after cardiac transplantation (late pneumonectomy) $(n=7)$. Control vs. early pneumonectomy $P<0.001$; control vs. late pneumonectomy $P<0.05$; early pneumonectomy vs. late pneumonectomy not significant. Histological appearance (H\&E) of (B) long-term-surviving BALB/c heart after transplantation into B6 mouse that carries a tolerant BALB/c lung allograft, (C) rejected BALB/c heart after transplantation into B6 mouse in which tolerant BALB/c lung allograft was removed 24 hours before heart transplantation, and (D) rejected BALB/C heart after transplantation into B6 mouse in which tolerant BALB/c lung allograft was removed 30 days after heart transplantation. Verhoeff's elastin stain of (E) long-term-surviving BALB/c heart after transplantation into B6 mouse that carries a tolerant BALB/C lung allograft, (F) rejected BALB/C heart after transplantation into B6 mouse in which tolerant BALB/c lung allograft was removed 24 hours before heart transplantation, and (G) rejected BALB/c heart after transplantation into B6 mouse in which tolerant BALB/c lung allograft was removed 30 days after heart transplantation. CTRL, control; PNX, pneumonectomy. Scale bars: $100 \mu \mathrm{m}$.

Our findings provide insights into the function of lymphatic vessels in tertiary lymphoid organs and may have important implications for a variety of disease processes.

\section{Results}

Induction and maintenance of peripheral tolerance after lung transplantation depends on the presence of the pulmonary allograft. We have previously reported that tolerance develops after lung transplantation, as evidenced by long-term acceptance of donor-matched, but not third-party, hearts by recipients of tolerant pulmonary allografts (12). We wanted to investigate whether the continued presence of the tolerant lung allograft was required for the induction of tolerance. To examine this, we transplanted $\mathrm{BALB} / \mathrm{c}$ lungs into $\mathrm{B} 6$ recipients that received perioperative costimulatory blockade, a regimen that we have previously shown results in tolerance induction after pulmonary transplantation (10). At least 30 days later, $\mathrm{BALB} / \mathrm{c}$ hearts were transplanted into these $\mathrm{B} 6$ hosts. When the lung allografts were removed 24 hours before engraftment of the heart, the cardiac grafts were rejected (Figure 1A). We observed intimal thickening and often luminal occlusion of coronary arteries in rejected grafts, consistent with the development of cardiac allograft vas- culopathy (Figure 1, B, C, E, and F). Next, we wanted to assess whether the presence of a tolerant pulmonary allograft was necessary to maintain peripheral tolerance. We therefore transplanted BALB/c hearts into B6 mice that had been engrafted with $\mathrm{BALB} / \mathrm{c}$ lungs at least 30 days prior while receiving perioperative costimulatory blockade. Thirty days after heart transplantation we removed the tolerant pulmonary allograft. While most hearts stopped beating, a few cardiac grafts continued to beat weakly during the observation period (Figure 1A). These grafts had evidence of cardiac allograft vasculopathy (Figure $1, \mathrm{D}$ and G). Compared with control grafts we observed a lower density of B cells, but a higher density of $\mathrm{T}$ lymphocytes in hearts that were rejected after either early or late removal of the tolerant lung allograft (Supplemental Figure 1, A-D; supplemental material available online with this article; https:// doi.org/10.1172/JCI136057DS1). Removal of the lung allograft before or after heart transplantation was also associated with a significantly lower cardiac allograft density of $\mathrm{Foxp}^{+}$cells as well as a decrease in the ratio of Foxp $3^{+}$cells to overall $\mathrm{T}$ lymphocytes (Supplemental Figure 1, E-G). Thus, donor-specific tolerance in the periphery cannot be induced or maintained in the absence of a tolerant lung allograft. 
A

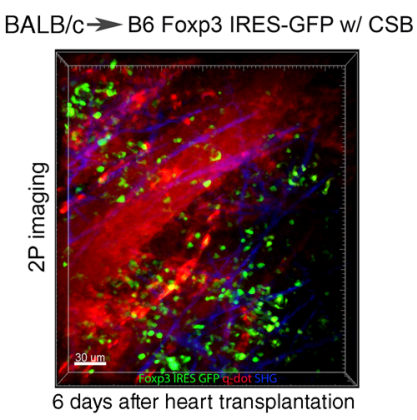

C

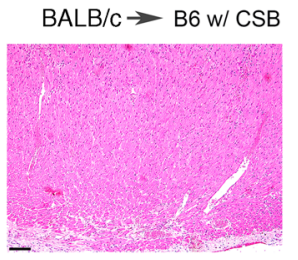

B

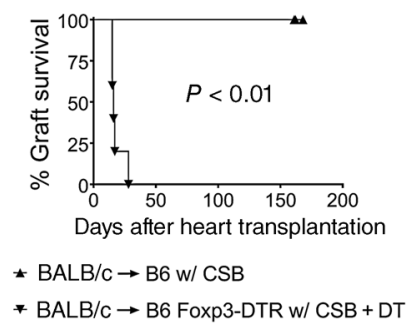

D
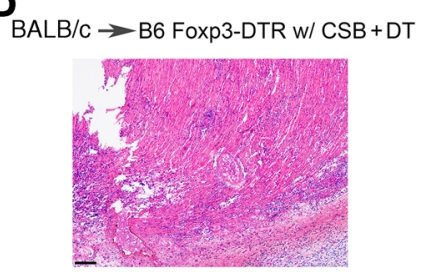

Figure 2. Foxp $3^{+}$cells are critical for long-term survival after heart transplantation. (A) Intravital 2-photon (2P) imaging depicting Foxp3 ${ }^{+}$ cells infiltrating BALB/c hearts 6 days after transplantation into B6 Foxp3 IRES-GFP mice (Foxp3 $3^{+}$cells: green; quantum dot-labeled [q-dotlabeled] vessels: red; second harmonic generation [SHC]: blue) $(n=3)$. (B) Kaplan-Meier survival curves of BALB/c hearts after transplantation into $(\boldsymbol{\Delta})$ B6 recipients $(n=5)$ or $(\boldsymbol{\nabla})$ DT-treated B6 Foxp3-DTR mice $(n$ = 5). Histological appearance $(H \& E)$ of (C) long-term-surviving BALB/C heart after transplantation into B6 recipient and (D) rejected heart after transplantation into DT-treated B6 Foxp3-DTR host. All recipients were treated with perioperative costimulatory blockade (CSB). Scale bars: 30 $\mu \mathrm{m}(\mathbf{A})$ and $100 \mu \mathrm{m}$ (C and D).

Immunoregulatory cells traffic from tolerant lung allograft to periphery during induction of tolerance. The dependence of peripheral tolerance on the presence of a tolerant pulmonary allograft pointed to a critical role for continuous trafficking of regulatory cells or secretion of soluble mediators from the transplanted lung. We have previously reported that bronchus-associated lymphoid tissue, a tertiary lymphoid organ, is induced in tolerant lung allografts (10). Furthermore, we have recently shown that graft-resident Foxp3 ${ }^{+}$ cells, which are located within the bronchus-associated lymphoid tissue, are critical to maintaining tolerance to transplanted lungs (12). To evaluate whether induction of heart transplant tolerance depends on Foxp $3^{+}$cells we transplanted BALB/c hearts into B6 Foxp3-GFP recipients (which express GFP under the control of the Foxp3 promoter) that received perioperative costimulatory blockade. Early after transplantation, we observed recruitment of recipient Foxp $3^{+}$cells to the cardiac graft where many Foxp $3^{+}$cells had extravasated (Figure 2A and Supplemental Video 1). More importantly, continuous depletion of recipient Foxp3 $3^{+}$cells after transplantation of BALB/c hearts into B6 Foxp3-DTR mice (which express the human diphtheria toxin receptor [DTR] under the control of the Foxp3 promoter) that received perioperative costimulatory blockade triggered the rejection of cardiac allografts (Figure 2, $\mathrm{B}-\mathrm{D})$. Thus, regulatory $\mathrm{T}$ cells are functionally important to mediate long-term survival of hearts in this model.

To examine whether Foxp $3^{+}$cells that reside in tolerant pulmonary allografts exit the lung and traffic to the periphery we

took advantage of our lung retransplant model. We have previously reported that tolerant lung allografts survive long term after retransplantation into nonimmunosuppressed recipients (10). We transplanted BALB/c lungs into B6 Foxp3-GFP recipients that received perioperative costimulatory blockade. At least 30 days later these tolerant grafts were retransplanted into nonimmunosuppressed B6 mice. Twenty-one days after lung retransplantation $\mathrm{BALB} / \mathrm{c}$ hearts were engrafted into these mice (Figure 3A). Six days after heart transplantation, we observed recruitment of Foxp3GFP cells to the transplanted hearts where many Foxp $3^{+}$cells had entered the graft tissue (Figure 3B and Supplemental Video 2). These findings indicated that Foxp $3^{+}$cells within the transplanted heart originated from the tolerant lung allograft. At this time point, we continued to detect $\mathrm{GFP}^{+}$cells in the lung graft (Figure $3 \mathrm{C}$ ). We observed that the majority of these cells expressed Ki-67 in cardiac allografts, which is consistent with their expansion. While we also observed their proliferation in lung allografts, the percentage of Ki-67-expressing cells was higher in the transplanted hearts (Supplemental Figure 2, A-C). These cells expressed high levels of intracellular CTLA-4 in both compartments (Supplemental Figure 2, D and E). Notably, such BALB/c heart allografts survived long term (Figure 3, D and E). In addition to Foxp $3^{+}$cells, we were able to detect other $\mathrm{T}$ lymphocyte populations, such as $\mathrm{CD} 8^{+} \mathrm{T}$ cells in transplanted hearts that have originated from the tolerant lung allografts. Most of these $\mathrm{CD} 8^{+} \mathrm{T}$ cells were $\mathrm{CD} 44^{\text {hi }} \mathrm{CD} 62^{\mathrm{lo}}$, consistent with an effector memory phenotype (Supplemental Figure 3, $\mathrm{A}$ and $\mathrm{B})$. Of note, while we observed that some $\mathrm{CD} 8^{+} \mathrm{T}$ cells in tolerant lung allografts expressed CD103, a marker that is characteristic of nonmigratory tissue-resident memory cells, CD103 expression was largely absent on $\mathrm{CD}^{+} \mathrm{T}$ cells in cardiac grafts that had originated from the lung grafts (13) (Supplemental Figure 3, C and D). In contrast to donor-matched BALB/c cardiac grafts, hearts derived from a third-party (CBA mice) were acutely rejected when transplanted into $\mathrm{B} 6$ recipients that had received tolerant BALB/c lung allografts 21 days prior (Supplemental Figure 4). We also assessed whether Foxp $3^{+}$cells migrate from tolerant BALB/c lung allografts into syngeneic B6 heart grafts. Only a small percentage of $\mathrm{CD}^{+} \mathrm{T}$ cells that were present in syngeneic $\mathrm{B} 6$ hearts 6 days after transplantation expressed Foxp3, very few of which were derived from the original host (Supplemental Figure 5).

Lymphatic drainage from bronchus-associated lymphoid tissue facilitates exit of Foxp $3^{+}$cells and mediates peripheral tolerance. Having shown that Foxp $3^{+}$cells exit the tolerant pulmonary allograft, we next set out to examine how cells migrate from the bronchusassociated lymphoid tissue in the transplanted lung to the periphery. In previous work we demonstrated that high endothelial venules, specialized vessels through which cells can enter secondary or tertiary lymphoid organs, form in bronchus-associated lymphoid tissue within tolerant lung allografts $(10,14)$. Consistent with previous reports that lymphatic vessels can also form in tertiary lymphoid organs, we observed many vascular structures that stained for VEGFR-3 within the bronchus-associated lymphoid tissues of tolerant lung allografts (Figure 4A) (3). We detected VEGFR-3 staining only in luminal cells. Interestingly, we observed Foxp $^{+}$cells within the lumina of these lymphatic vessels.

Peribronchial lymphatic vessels are severed as part of the lung transplant procedure. To evaluate at what time point after lung 
A BALB/clung B6/B6 Foxp3 IRES-GFP

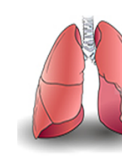

B BALB/c lung $\rightarrow$ B6 Foxp3 IRES-GFP(CSB 30 d) $\rightarrow$ B6 $(\geq 21$ d)
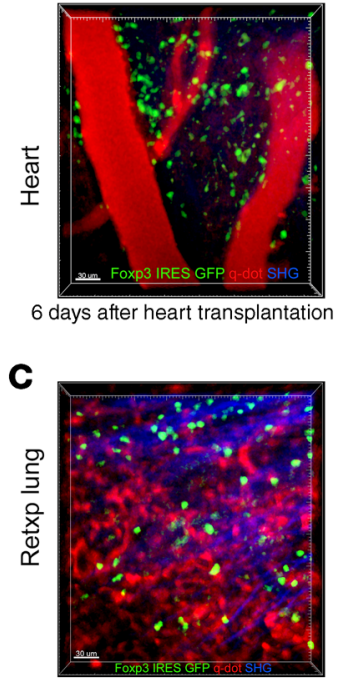

B6

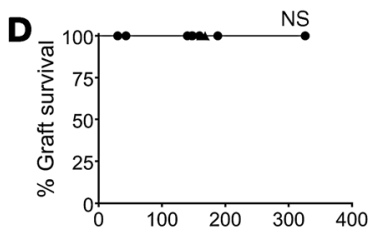

Days after heart transplantation

$\star \mathrm{BALB} / \mathrm{c} \rightarrow \mathrm{B} 6 \mathrm{w} / \mathrm{CSB}$

- BALB/c $\rightarrow$ B6 (Retxp lung $\geq 21 \mathrm{~d}$ )

$B A L B / c$ heart

\section{$\mathbf{E}$}

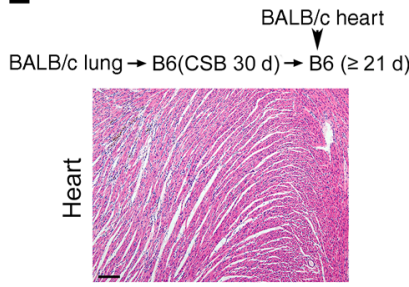

transplantation lymphatic drainage from the pulmonary graft to the periphery is reestablished, we transplanted BALB/c lungs into B6 mice that received perioperative costimulatory blockade. Lymphatic drainage from the lung allograft was evaluated 21 days after transplantation by instilling a fluorescent dye into the bronchus of the graft. We observed accumulation of fluorescent dye in the draining mediastinal lymph nodes, albeit at somewhat lower levels when compared with naive nontransplanted mice (Figure 4B). However,
Figure 3. Peripheral tolerance is associated with exit of Foxp $3^{+}$cells from tolerant lung allograft. (A) Schematic diagram depicting experimental transplant model. Intravital 2-photon imaging of (B) BALB/C cardiac allograft $(n=3)$ and (C) retransplanted (Retxp) tolerant BALB/c lung allograft $(n=3) 6$ days after transplantation of BALB/c heart into B6 mouse into which a tolerant BALB/c lung was retransplanted at least 21 days prior (Foxp $3^{+}$cells: green; quantum dot-labeled [q-dot-labeled] vessels: red; second harmonic generation [SHG]: blue). The BALB/c lung had been originally transplanted into a B6 Foxp3 IRES-GFP mouse (treated with perioperative costimulatory blockade) at least 30 days before the retransplant procedure. (D) Kaplan-Meier survival curves of BALB/c hearts $(\boldsymbol{\Delta})$ that were transplanted into $\mathrm{B} 6$ recipients that received perioperative costimulatory blockade $(n=5)$ or $(\bullet)$ that were transplanted into nonimmunosuppressed B6 mice that received tolerant BALB/c pulmonary allografts at least 21 days before cardiac transplantation $(n=7)$. The $B A L B / c$ lungs had been originally engrafted into B6 mice that received perioperative costimulatory blockade and then retransplanted at least 30 days later. (E) Histological appearance (H\&E) of long-term-surviving $\mathrm{BALB} / \mathrm{c}$ hearts after transplantation into B6 mice into which a tolerant $B A L B / c$ lung allograft was retransplanted at least 21 days prior. Scale bars: $30 \mu \mathrm{m}$ (B and C) and $100 \mu \mathrm{m}$ (E).
A

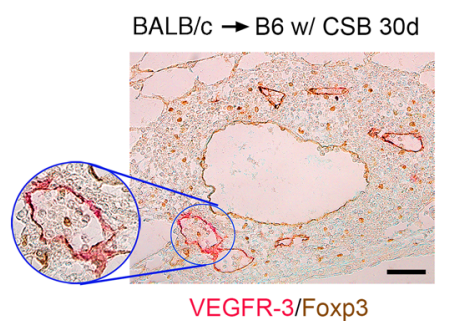

VEGFR-3/Foxp3
B

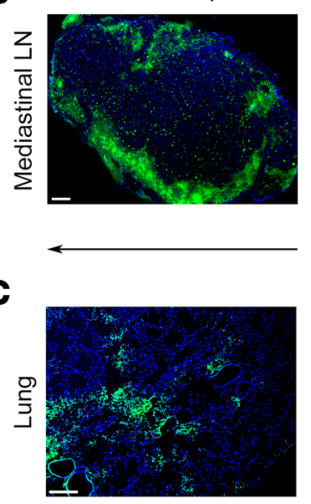

$\operatorname{Txp} 7 d$

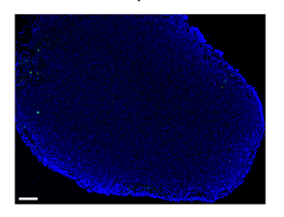

Dextran Alexa-488/DAPI

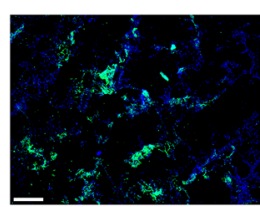

when we administered fluorescent dye into the graft bronchus 7 days after transplantation, there was virtually no detectable dye in the draining lymph nodes (Figure 4B). These findings are consistent with previously published reports in large animals and more recently in mice demonstrating that lymphatic drainage from pulmonary grafts is reestablished approximately 2 to 3 weeks after the lung transplant procedure $(15,16)$. No differences in the density of the fluorescent dye were detected in the lungs among these conditions (Figure 4C).

Figure 4. Lymphatic drainage from lung allograft is reestablished within 21 days after transplantation. (A) Immunostaining of VEGFR-3 and Foxp3 in bronchus-associated lymphoid tissue in BALB/c lung 30 days after transplantation into B6 recipient that was treated with costimulatory blockade. Representative images and quantification of intensity of fluorescent dye (dextran-Alexa Fluor 488) in (B) draining mediastinal lymph nodes (LN) (original magnification, $\times 100$ ) and (C) left lungs (original magnification, $\times 50$ ) in naive B6 mice, B6 mice 7 days or 21 days after transplantation (Txp) of BALB/C lungs (DAPI blue) $(n=5)$. Lung recipients were treated with perioperative costimulatory blockade. Scale bars: $100 \mu \mathrm{m}(\mathbf{A}$ and $\mathbf{B})$ and $200 \mu \mathrm{m}(\mathbf{C})$. Data are expressed as mean \pm SEM. One-way ANOVA was used to compare the means. NS, not significant. 
A
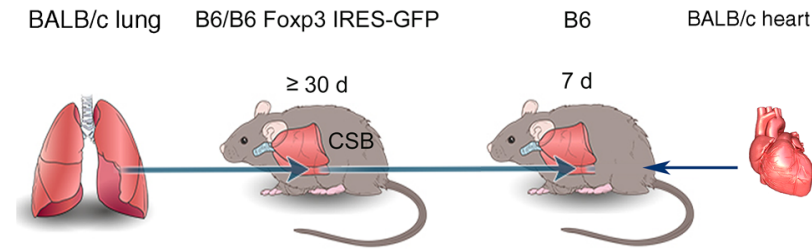

B

BALB/c heart

BALB/c lung $\rightarrow$ B6 Foxp3 IRES-GFP $($ CSB 30d) $\rightarrow$ B6 (7d)

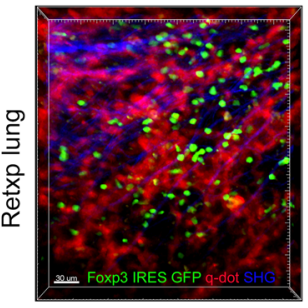

C

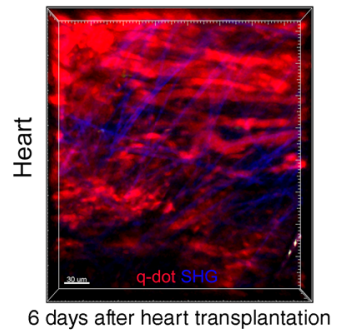

Figure 5. Peripheral tolerance is not induced in the absence of lymphatic drainage from the tolerant lung allograft. (A) Schematic diagram depicting experimental transplant model. Intravital 2-photon imaging of (B) retransplanted (Retxp) tolerant BALB/C lung allograft $(n=3)$ and (C) BALB/c cardiac allograft $(n=3) 6$ days after transplantation of $B A L B / C$ heart into $B 6$ mouse into which a tolerant BALB/c lung was retransplanted 7 days prior (Foxp3 ${ }^{+}$cells: green; quantum dot-labeled [q-dot-labeled] vessels: red; second harmonic generation [SHC]: blue). The BALB/c lung had been transplanted into a B6 Foxp3 IRES-GFP mouse (treated with perioperative costimulatory blockade) at least 30 days before the retransplant procedure. (D) Kaplan-Meier survival curves of BALB/C hearts that were transplanted into nonimmunosuppressed $B 6$ mice that received tolerant BALB/c pulmonary allografts $(\bullet) 7(n=8)$ or $(\boldsymbol{\Delta})$ at least 21 days $(n=$ 7) before cardiac transplantation. The BALB/c lungs had been originally engrafted into B6 mice that received perioperative costimulatory blockade and then retransplanted at least 30 days later. $(\boldsymbol{\nabla})$ Depicts the survival of BALB/c hearts that were transplanted into DT-treated B6 Foxp3-DTR mice that received perioperative costimulatory blockade. (E) Histological appearance (H\&E) of rejected BALB/c hearts after transplantation into B6 mice into which a tolerant BALB/c lung allograft was retransplanted 7 days prior. Scale bars: $30 \mu \mathrm{m}$ (B and C) and $100 \mu \mathrm{m}$ (E).

Next, we wanted to evaluate whether the reestablishment of lymphatic drainage from the tolerant lung allograft was critical for the induction of peripheral tolerance. To this end, we transplanted BALB/c lungs into B6 Foxp3-GFP recipients that received perioperative costimulatory blockade. At least 30 days later these tolerant grafts were retransplanted into nonimmunosuppressed B6 mice. Seven days after lung retransplantation $\mathrm{BALB} / \mathrm{c}$ hearts were engrafted into these mice (Figure $5 \mathrm{~A}$ ). Although Foxp3-GFP cells were present in the lungs, we did not observe Foxp3-GFP cells within the cardiac allografts 6 days after heart transplantation (Figure 5, B and C). In contrast to the longterm survival of cardiac allografts that were engrafted 21 days after lung retransplantation, hearts that were transplanted 7 days after lung retransplantation were rejected (Figure 5, D and E). We next wanted to determine if pharmacologically induced lymphan- giogenesis could facilitate peripheral tolerance in this model. Here, we employed VEGF-C156S, a VEGF-C mutant with exclusive binding affinity for VEGFR-3, a receptor that is expressed on lymphatic endothelial cells and mediates lymphatic sprouting (17). Although VEGFR-3 expression has been observed by immunostaining on sprouting blood vessels in monkeys and humans, most studies indicate that VEGF-C156S does not promote angiogenesis in adults (18-21). Given that our vascularized lung transplant model establishes blood flow at the time of engraftment, any potential effect of VEGF-C156S on new blood vessel formation is not likely to impact cell migration. Also, VEGF-C156S did not increase the chemotaxis of regulatory $\mathrm{T}$ cells compared with vehicle control (Supplemental Figure 6). We treated B6 recipients with VEGF-C156S for 7 days after receiving a tolerant $\mathrm{BALB} / \mathrm{c}$ lung allograft and then transplanted BALB/c hearts into these mice. In contrast to our observations without VEGF-C156S treatment (Figure 5C), administration of VEGF-C156S resulted in the infiltration of lung allograft-derived Foxp3-GFP cells into the cardiac allografts (Figure 6, $\left.\mathrm{A}^{-} \mathrm{C}\right)$, which was associated with their long-term survival (Figure 6, D and E).

Finally, we wanted to evaluate whether disruption of lymphatic growth prevented induction of peripheral tolerance after lung transplantation. Here, we employed an anti-VEGFR-3 antibody that we and others have shown to inhibit lymphatic regrowth $(22,23)$. We transplanted $\mathrm{BALB} / \mathrm{c}$ lungs into $\mathrm{B} 6$ Foxp3-GFP recipients that received perioperative costimulatory blockade. At least 30 days later these tolerant lungs were retransplanted into nonimmunosuppressed B6 hosts that received treatment with anti-VEGFR-3. Twenty-one days later we transplanted BALB/c hearts into these mice. While Foxp3-GFP cells were present in the lung allografts, in contrast to control conditions (Figure 3B), we did not observe $\mathrm{GFP}^{+}$cells in the cardiac allografts 6 days after heart transplantation (Figure 7, A-C). All hearts stopped beating, with histological evidence of rejection (Figure 7, D and E), similarly to heart grafts implanted 7 days after lung retransplantation (Figure 5, D and E). The lung allografts did not have evidence of rejection (Supplemental Figure 7, A and B). However, the size of their bronchus-associated lymphoid tissue was increased compared with mice that did not receive treatment with anti-VEGFR-3 (Supplemental Figure 7C). Moreover, many lymphatic vessels were filled with cells, including Foxp ${ }^{+}$cells (Supplemental Figure 7D).

\section{Discussion}

In this study, we took advantage of a temporal requirement for the reestablishment of lymphatic drainage from lungs after transplantation to show that immune cells exit tolerant pulmonary grafts via lymphatics. Retransplantation of tolerant lung allografts has allowed us to uncover that efferent lymphatic flow from tertiary lymphoid organs can regulate immune responses in the periphery. We show that long-term survival of cardiac 


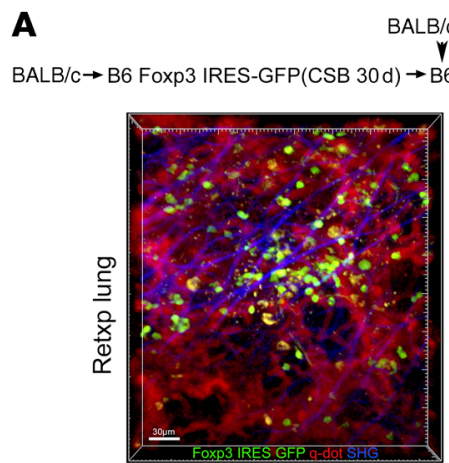

$\mathrm{ALB} / \mathrm{c}$ heart

6 (VEGF-C156S 7 d)

B

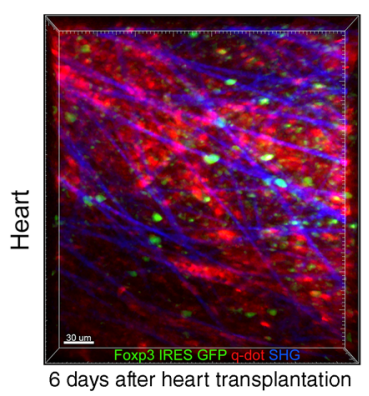

6 days after heart transplantation
C

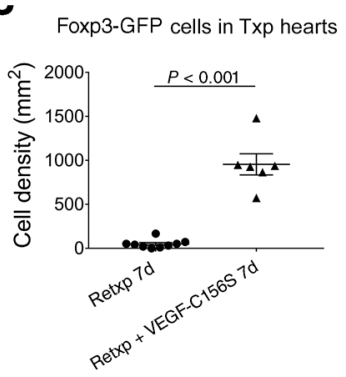

D

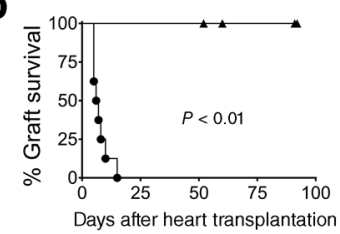

- BALB/C $\rightarrow$ B6 (Retxp lung 7d)

$\star B A L B / c \rightarrow B 6$ (Retxp lung + VEGF-C156S 7d)

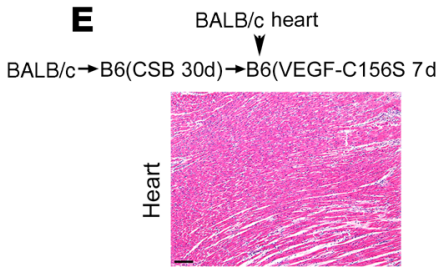

Figure 6. Stimulation of lymphangiogenesis promotes induction of peripheral tolerance after lung transplantation. Intravital 2-photon imaging of (A) retransplanted (Retxp) tolerant BALB/c lung allograft $(n=2)$ and (B) BALB/c cardiac allograft $(n=2) 6$ days after transplantation of $B A L B / c$ heart into a $B 6$ mouse into which a tolerant BALB/c lung was retransplanted 7 days prior and received VEGF-C156S between the time of lung retransplantation and heart transplantation (Foxp3 ${ }^{+}$cells: green; quantum dot-labeled [q-dot-labeled] vessels: red; second harmonic generation [SHG]: blue). The BALB/c lung had been originally transplanted into a B6 Foxp3 IRES-GFP mouse (treated with perioperative costimulatory blockade [CSB]) at least 30 days before the retransplant procedure. (C) Quantification of density of Foxp3-GFP cells in $\mathrm{BALB} / \mathrm{c}$ hearts (up to 5 separate areas/graft were examined) 6 days after transplantation (Txp) into $B 6$ recipients of tolerant BALB/c lung allografts, that received no treatment or were treated with VEGF-C156S for 7 days before cardiac transplantation. Data are expressed as mean \pm SEM. Mann-Whitney $U$ test was used to compare the means. (D) Kaplan-Meier survival curves of BALB/c hearts that were transplanted into nonimmunosuppressed B6 mice, $(\bullet)$ untreated $(n=7)$ or $(\mathbf{\Delta})$ treated with VECF-C156S $(n=4)$, that received tolerant BALB/c pulmonary allografts 7 days before cardiac transplantation. The BALB/c lungs had been originally engrafted into $\mathrm{B} 6$ mice that received perioperative costimulatory blockade and then retransplanted at least 30 days later. (E) Histological appearance (H\&E) of long-term-surviving BALB/c hearts after transplantation into VEGFC-156S-treated B6 mice into which a tolerant BALB/C lung allograft was retransplanted 7 days prior. Scale bars: $30 \mu \mathrm{m}$ (A and $\mathbf{B})$ and $100 \mu \mathrm{m}(\mathbf{E})$.

ies have linked lymphangiogenesis in murine and human allografts, irrespective of their association with tertiary lymphoid organs, to the propagation of immune responses that mediate rejection (2527). Lymphatic vessel density is increased in the myocardium of rat cardiac allografts that undergo chronic rejection (28). Interestingly, inhibition of VEGFR-3 resulted in prolongation of rat heart allograft survival when recipients received immunosuppression with low-dose cyclosporine, which may in part be related to decreased trafficking of antigen-presenting cells via lymphatic vessels from the graft to the draining secondary lymphoid organs. Similar roles have been proposed for lymphatic vessels in islet, tracheal, and corneal allografts, where anti-VEGFR-3-mediated inhibition of lymphangiogenesis results in attenuation of the immune response and prevention of tissue destruction (29-31).

However, evidence has emerged that lymphangiogenesis in allografts is not necessarily harmful and may in fact promote the resolution of inflammation. Lungs differ from other organ and tissue grafts, as alloimmune responses that regulate rejection and tolerance occur locally within the pulmonary graft and are not dependent on cellular trafficking to secondary lymphoid organs $(10,32)$. Thus, unlike the case for hearts, islets, and corneas, we have shown that lymphatic migration of antigen-presenting cells from pulmonary grafts to the periphery is not critical for graft rejection (33-35). In fact, promoting lymphangiogenesis through treatment with VEGF-C has been shown to attenuate acute rejection of mouse lung allografts (19). Mechanistically, lymphatic vessels facilitate the clearance of damage-associated molecular patterns, such as hyaluronan fragments that are known to accentuate inflammatory responses following lung transplantation through engagement of Toll-like receptors (36). These findings are somewhat reminiscent of previous studies that demonstrated that VEGF-C-driven activation of lymphatic vessels limits inflammation of the skin $(18,37)$. A more recent study showed that inducallografts depends on Foxp $3^{+}$cells and that the survival of donormatched hearts is associated with their infiltration with Foxp $3^{+}$ cells that originate from tolerant lung allografts. In this setting, peripheral tolerance cannot be induced in the absence of lymphatic drainage from the lung allograft.

Most tertiary lymphoid organs in transplanted tissues or organs have been associated with the promotion of immune responses that are deleterious to the graft. For example, tertiary lymphoid organs have been observed in mouse cardiac allografts that have evidence of acute or chronic rejection (9). Moreover, lymphoid neogenesis in rat aortic allografts has been shown to contribute to the generation of a local humoral alloimmune response (24). Similarly, most stud- tion of lymphangiogenesis in murine renal allografts through doxycycline-induced expression of VEGF-C resulted in prolongation of their survival (38). Although the underlying mechanism was not explored, the authors speculated that lymphatic endothelial cells may facilitate the generation or trafficking of immunoregulatory cells. In support of this, evidence exists that lymphatic endothelial cells can suppress $\mathrm{T}$ cell responses directly by presenting antigen in tolerogenic fashion or indirectly by inhibiting the maturation of dendritic cells (39).

We have not observed the induction of bronchus-associated lymphoid tissue in long-term-surviving syngeneic lung grafts, indicating that severing lymphatic connections between the graft and 


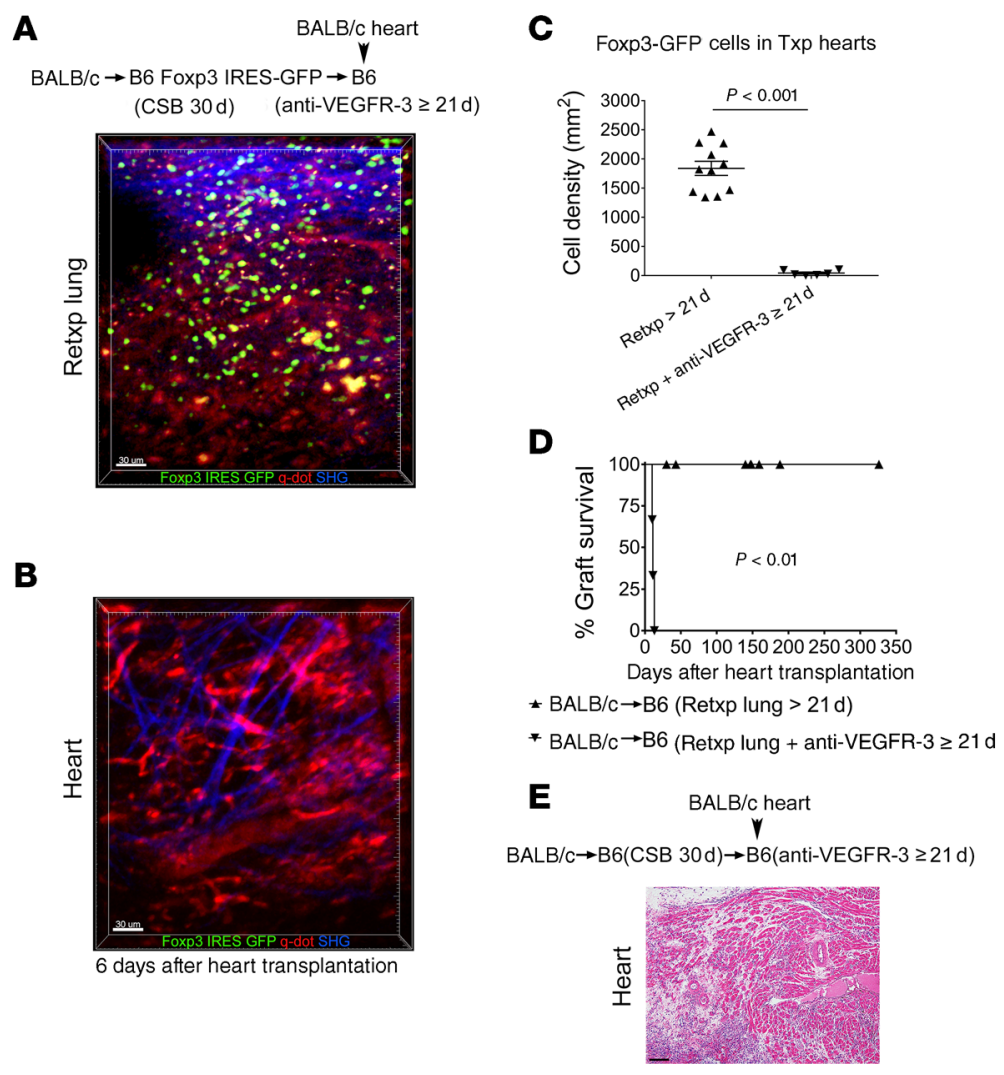

Figure 7. Inhibition of lymphatic regrowth after lung transplantation prevents induction of peripheral tolerance. Intravital 2-photon imaging of $(A)$ retransplanted (Retxp) tolerant BALB/c lung allograft $(n=2)$ and (B) BALB/c cardiac allograft $(n=2)$ 6 days after transplantation of BALB/c heart into a B6 mouse into which a tolerant $B A L B / C$ lung was retransplanted 21 days prior and received anti-VEGFR-3 between the time of lung retransplantation and heart transplantation (Foxp3 ${ }^{+}$cells: green; quantum dot-labeled [q-dot-labeled] vessels: red; second harmonic generation [SHG]: blue). The $\mathrm{BALB} / \mathrm{C}$ lung had been originally transplanted into a B6 Foxp3 IRES-GFP mouse (treated with perioperative costimulatory blockade [CSB]) at least 30 days before the retransplant procedure. (C) Quantification of density of Foxp3-GFP cells in BALB/c hearts (up to 5 separate areas/graft were examined) 6 days after transplantation (Txp) into B6 recipients of tolerant $B A L B / c$ lung allografts that received no treatment or were treated with anti-VEGFR-3 for 21 days before cardiac transplantation. Data are expressed as mean \pm SEM. Mann-Whitney $U$ test was used to compare the means. (D) Kaplan-Meier survival curves of BALB/C hearts that were transplanted into nonimmunosuppressed B6 mice, $(\bullet)$ untreated $(n=7)$ or $(\boldsymbol{\Delta})$ treated with anti-VEGFR-3 $(n=3)$, that received tolerant $\mathrm{BALB} / \mathrm{c}$ pulmonary allografts 21 days before cardiac transplantation. The $B A L B / c$ lungs had been originally engrafted into $B 6$ mice that received perioperative costimulatory blockade and then retransplanted at least 30 days later. (E) Histological appearance (H\&E) of rejected BALB/c hearts after transplantation into anti-VEGFR-3treated $\mathrm{B} 6$ mice into which a tolerant $\mathrm{BALB} / \mathrm{c}$ lung allograft was retransplanted 21 days prior. Scale bars: $30 \mu \mathrm{m}$ (A and B) and $100 \mu \mathrm{m}(\mathbf{E})$.

the recipient as part of the surgical procedure is not sufficient to induce tertiary lymphoid organs (10). Thus, in the setting of lung transplantation, the continuous presence of alloantigen in the graft appears to be a prerequisite for the induction of bronchus-associated lymphoid tissue. A recent study, however, showed that inducible genetic ablation of lymphatic endothelial cells in syngeneic lung grafts resulted in the induction of tertiary lymphoid organs (40). Of note, lymphatic endothelial cells in the grafts were ablated 21 days after lung transplantation to allow for regrowth of lymphatic vessels at the pulmonary hilum. It is important to note that existing pulmonary lymphatic vessels were destroyed in this study, which likely accounts for the drastic differences in phenotype compared with transient interruption of lymphatic flow after syngeneic lung transplantation in our previous experiments (10). It is noteworthy that treatment with anti-VEGFR-3, a regimen that we and others have shown inhibits the growth of new lymphatic vessels, results in an increase in the size of the bronchus-associated lymphoid tissue in lung allografts $(22,41)$. This finding, combined with our observation that many lymphatic vessels in the bronchus-associated lymphoid tissue are filled with lymphocytes after administration of anti-VEGFR-3, lends further support to the notion that these lymphatics provide flow to the periphery. In support of this, we have shown that, in addition to Foxp $3^{+}$cells, other immune cells, such as $\mathrm{CD}^{+} \mathrm{T}$ lymphocytes, can also migrate from tolerant lung allografts to the periphery.

Foxp $^{+}$cells play an important role in mediating transplantation tolerance. Consistent with our observations, previous experimental studies have shown that Foxp $3^{+}$cells home to allografts during the induction of tolerance. Also, positive correlations between the presence of Foxp $3^{+}$cells in murine and human allografts and graft survival or function have been reported $(42,43)$. Our observations extend a previous report that demonstrated the presence of regulatory $\mathrm{CD} 4^{+} \mathrm{T}$ cells in tolerant murine skin grafts (44). Interestingly, retransplantation of tolerant skin grafts onto immunodeficient mice revealed that $\mathrm{T}$ cells can exit the graft and suppress alloantigen-specific immune responses in the periphery. Although migratory requirements of $\mathrm{T}$ lymphocytes were not directly examined, it is likely that immune cells exit skin allografts through afferent lymphatics (45). Our finding that the presence of a tolerant lung allograft is critical to induce tolerance to donor-matched hearts is somewhat reminiscent of previous observations in miniature swine kidney transplant and murine heart transplant models (46, 47). However, in these models, the time taken to lose tolerance after removal of the primary graft was substantially longer compared with our study. We suggest that the continuous presence of donor alloantigen within the bronchus-associated lymphoid tissue of tolerant lung allografts is necessary for the expansion, survival, and/or activation of Foxp $3^{+}$cells. We have previously reported that recipient Foxp $3^{+}$cells accumulate in tolerant lung allografts following retransplantation into nonimmunosuppressed hosts. This raises the possibility that Foxp $3^{+}$cells that reside within tertiary lymphoid organs of tolerant lung allografts may be able to confer regulatory properties upon other $\mathrm{T}$ cells, a process referred to as infectious tolerance $(48,49)$. Thus, while it is conceivable that Foxp $3^{+}$ cells that originate from tolerant lung allografts promote infectious tolerance in the periphery, the rejection of heart grafts after anti-VEGFR-3 antibody-mediated inhibition of lymphatic regrowth and the rejection of third-party grafts demonstrate that 
recipient regulatory $\mathrm{T}$ cells alone are not sufficient to mediate the survival of heart grafts.

We have recently reported that IL-22 production by innate lymphoid cells type 3 and $\gamma \delta$ T cells plays a critical role in inducing organized bronchus-associated lymphoid tissue in tolerant lung allografts (14). In the absence of IL-22, Foxp3-rich lymphoid follicles are induced in lung allografts, but B cells are not recruited to these structures and peripheral nodal addressin-expressing high endothelial venules are not formed. However, VEGFR $-3^{+}$lymphatic vessels develop in proximity to the Foxp $3^{+}$aggregates (data not shown), indicating that induction of high endothelial venules and lymphangiogenesis are regulated through different mechanisms. Furthermore, IL-22-deficient lung allograft recipients accept donor-matched cardiac allografts long term, indicating that high endothelial venules within the transplanted lungs are not necessary to mediate peripheral tolerance (data not shown). Several cell populations, including dendritic cells and macrophages, as well as soluble mediators such as IL-17 or VEGF-C have been suggested to play a role in the regulation of lymphangiogenesis during inflammation (50-53). As cellular and molecular requirements that regulate the induction of tertiary lymphoid organs may depend on the anatomical site and the nature of the inflammatory signal, future studies will need to determine how lymphangiogenesis is induced in the bronchus-associated lymphoid tissue in tolerant lung allografts (54-56).

Lymphangiogenesis has been reported in tertiary lymphoid organs surrounding tumors. These lymphatic vessels can provide avenues for metastatic spread of cancer cells. Interestingly, recent work has shown that, similar to our observations in tolerant lung allografts, Foxp3-rich tertiary lymphoid organs can also form in association with tumors (57). Therefore, our findings raise the possibility that growth of metastatic tumor deposits may be facilitated by lymphatic migration of Foxp $3^{+}$cells that recognize tumor antigens from the primary tumor site to the periphery. Similarly, one can envision that effector $\mathrm{T}$ cells may leave tertiary lymphoid organs that are induced in the setting of cancer or autoimmune disease and promote cytotoxic or inflammatory responses at other sites (58). Future studies will need to elucidate the chemotactic cues that promote lymphatic egress of immune cells from tertiary lymphoid organs.

\section{Methods}

Mice and reagents. C57BL/6 (B6), B6 CD45.1, BALB/c, CBA, B6 Foxp3-DTR, and B6 Foxp3-IRES-GFP mice were purchased from The Jackson Laboratory. As indicated, select lung and heart transplant recipients were treated with perioperative costimulatory blockade consisting of anti-CD40 ligand $(250 \mu \mathrm{g}$ intraperitoneally [i.p.], day 0) and CTLA-4-Ig (200 $\mu$ g i.p., day 2) (Bio X Cell). Diphtheria toxin (DT) (List Biological Laboratories Inc.) (500 ng i.p. days 0,1 , and 7) was given to select B6 Foxp3-DTR heart transplant recipients to deplete Foxp $3^{+}$cells. Select lung retransplant recipients received treatment with anti-VEGFR-3 antibody (clone $\mathrm{mF} 4-31 \mathrm{C} 1$ ) (provided by Eli Lilly) (60 mg/kg, i.p. day 0; $30 \mathrm{mg} / \mathrm{kg}$ day 2; and then q.o.d. until day 21) or VEGF-C156S (R\&D Systems) $(125 \mu \mathrm{g} / \mathrm{kg}$, intravenously q.d. for 7 days). Six- to 10 -week-old mice were used for these studies. Gender-matched mice were used for the transplant procedures.
Surgical procedures. Orthotopic vascularized left lung transplants and retransplants, cervical heart transplants, and pneumonectomies were performed as previously described $(10,59-61)$. Cardiac graft survival was determined by cessation of a palpable heartbeat, which was confirmed by direct visualization.

Lung lymphatic drainage assessment. Mice were anesthetized and an angiocatheter $(24 \mathrm{G})$ was placed into left main bronchus. DextranAlexa Fluor $488(30 \mu \mathrm{L})$ solution $(5 \mu \mathrm{g} / \mu \mathrm{L}$ diluted in PBS; Life Technologies) was administered into the left main bronchus through the catheter following the inflation of the left lung (62). Left lungs and mediastinal lymph nodes were harvested 50 minutes later. The lungs were inflated with a 1:1 mixture of NEG-50 (Thermo Fisher Scientific) and PBS. Samples were then embedded in NEG-50 and solidified on dry ice. Frozen 10- $\mu$ m sections were cut using a cryostat (Leica CM1900), mounted on positively charged slides, and fixed with $4 \%$ paraformaldehyde in PBS.

Histology and immunostaining. Lung and heart allografts were fixed in formaldehyde, sectioned, and stained with hematoxylin and eosin (H\&E) or Verhoeff's elastin stain. The size of the bronchusassociated lymphoid tissue was quantitatively analyzed using ImageJ image processing and analysis software (NIH). For immunohistochemistry, formalin-fixed, 5 - $\mu$ m sections of paraffin-embedded specimens were deparaffinized and rehydrated. Following antigen retrieval in citrate buffer ( $\mathrm{pH}$ 6.0, Dako), endogenous peroxide activity was quenched with $3 \% \mathrm{H}_{2} \mathrm{O}_{2}$. The slides were washed in PBS with $1.5 \%$ bovine serum albumin (BSA) and 0.1\% Tween 20 (PBT). Nonspecific immunobinding sites were blocked with serum-free protein block (Dako) and sequential incubation in Avidin D and biotin-blocking solution (Vector Laboratories). Sections were incubated with primary antibodies overnight at $4^{\circ} \mathrm{C}$ in a humidified chamber. The primary antibodies used were goat polyclonal anti-mouse VEGFR-3 (R\&D Systems), rabbit monoclonal anti-CD3\& (clone SP7, Thermo Fisher Scientific), rat monoclonal anti-B220 (clone RA3-6B2, Thermo Fisher Scientific), and rabbit polyclonal anti-Foxp3 (ab75763, Abcam). Following primary antibody labeling slides were washed in PBT and then incubated with secondary horse anti-goat IgG, goat anti-rat IgG, or horse anti-rabbit IgG (Vector Laboratories), as indicated. Densities of

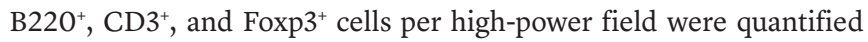
with ImageJ software, as previously described (14).

Two-photon microscopy. Intravital 2-photon microscopy of lung or heart grafts was performed for up to 3 hours, as previously described $(60,63)$. Quantum dots $(655 \mathrm{~nm}, 30 \mu \mathrm{L})$ (Life Technologies) were injected intravenously immediately before imaging to label vessels. Grafts were exposed and imaged with a custom 2-photon microscope using ImageWarp (A\&B Software). Sequential Z-sections $(2.5 \mu \mathrm{m}$ each) were acquired, yielding an imaging volume of $220 \times 247.5 \times 50$ $\mu \mathrm{m}^{3}$. Analysis of cell densities was performed in up to 5 heart areas per mouse with Imaris (Bitplane).

Flow cytometry. Heart and lung grafts were processed for flow cytometric staining as previously described (12). Antibodies used for surface and intracellular staining included anti-CD45.1 (clone A20, BD Biosciences), anti-CD45.2 (clone 104, Thermo Fisher Scientific), anti-CD90.2 (clone 53-2.1, Thermo Fisher Scientific), anti-CD4 (clone RM4-5, BioLegend), anti-CD8 $\alpha$ (clone 53.6.7, Thermo Fisher Scientific), anti-CD44 (clone IM7, eBioscience), anti-CD62L (clone MEL-14, BioLegend), anti-CD103 (clone 2E7, BioLegend), Foxp3 (clone FJK16s, Thermo Fisher Scientific), anti-Ki-67 (clone SolA15, Thermo 
Fisher Scientific), anti-CTLA-4 (clone UC10-4F10-11, BD Biosciences), and isotype controls (BD Biosciences, eBioscience). Intracellular staining (Ki-67, CTLA-4) was performed as previously described (12). Samples were acquired on an LSR Fortessa X-20 equipped with 5 lasers (355, 405, 488, 561, and $640 \mathrm{~nm}$; BD Biosciences) and analyzed using FlowJo version 10.5 (Tree Star).

Chemotaxis assay. $\mathrm{CD} 4^{+} \mathrm{T}$ cells were isolated from spleens and lymph nodes of $\mathrm{B} 6$ mice with a naive $\mathrm{CD} 4^{+} \mathrm{T}$ cell isolation kit (Miltenyi Biotec). To enrich for Foxp $3^{+}$cells, they were resuspended in $1 \mathrm{~mL}$ of RPMI 1640 medium, supplemented with 10\% FBS, TGF- $\beta$ ( $2 \mathrm{ng} / \mathrm{mL}$ ) (BioLegend), anti-mouse CD28 antibody $(2 \mu \mathrm{g} / \mathrm{mL})$ (BioLegend), and mouse recombinant IL-2 $(10 \mathrm{ng} / \mathrm{mL})$ (BioLegend) at a concentration of $2 \times 10^{6}$ cells $/ \mathrm{mL}$. This cell suspension was added at $1 \mathrm{~mL} /$ well to 24 -well plates that had been coated with anti-mouse CD3 $\varepsilon$ antibody (BioLegend) at $4^{\circ} \mathrm{C}$ overnight. The plates were washed with PBS before adding the $\mathrm{CD} 4^{+} \mathrm{T}$ cells. Four days later, $\mathrm{CD}^{+} \mathrm{T}$ cells were harvested and $1 \times 10^{5}$ cells in $100 \mu \mathrm{L}$ media were placed in $6.5-\mathrm{mm}$ inserts with $3.0-\mu \mathrm{m}$ polyester membranes in 24-well plates (Corning). Media $(600 \mu \mathrm{L})$ containing CCL22 (10 ng/ $\mathrm{mL}$ ) (R\&D Systems) or VEGF-C156S (1 or $10 \mathrm{ng} / \mathrm{mL}$ ) (R\&D Systems) or control media were added to the bottom wells. Cells that had migrated to the bottom wells were counted 4 and 21 hours later.

Statistics. Data were analyzed with the Mann-Whitney $U$ test or 1-way ANOVA (Prism, version 7.0; GraphPad Software). Data were graphed as mean \pm SEM. Heart allograft survival was compared between groups with Kaplan-Meier analysis. Differences were considered statistically significant at $P$ less than 0.05 .
Study approval. Animal experiments were approved by the Institutional Animal Care and Use Committee at Washington University. Animals received humane care in compliance with the Guide for the Care and Use of Laboratory Animals (National Academies Press, 2011) and with the Principles of Laboratory Animal Care formulated by the National Society for Medical Research.

\section{Author contributions}

WL, JMG, AYT, and RH conducted experiments. WL, JMG, AYT, YT, RH, CCF, MSH, KH, AIB, JHR, RGN, VP, BWW, KJL, AB, ASK, and AEG contributed to study design and reviewed and revised the manuscript. WL, JMG, AYT, and DK analyzed and interpreted the data. WL, JMG, and DK wrote the manuscript. DK designed and supervised the study.

\section{Acknowledgments}

DK is supported by NIH grants 1P01AI116501, R01HL094601, R01HL151078, Veterans Administration Merit Review grant 1I01BX002730, The Cystic Fibrosis Foundation, and The Foundation for Barnes-Jewish Hospital.

Address correspondence to: Daniel Kreisel, Professor of Surgery, Pathology \& Immunology, Campus Box 8234, 660 South Euclid Avenue, Washington University School of Medicine in St. Louis, St. Louis, Missouri 63110, USA. Phone: 314.362.6021; Email: kreiseld@wustl.edu.
1. Marin ND, Dunlap MD, Kaushal D, Khader SA. Friend or foe: the protective and pathological roles of inducible bronchus-associated lymphoid tissue in pulmonary diseases. J Immunol. 2019;202(9):2519-2526.

2. Jones E, Gallimore A, Ager A. Defining high endothelial venules and tertiary lymphoid structures in cancer. Methods Mol Biol. 2018;1845:99-118.

3. Baluk P, et al. Preferential lymphatic growth in bronchus-associated lymphoid tissue in sustained lung inflammation. Am J Pathol. 2014;184(5):1577-1592.

4. Rangel-Moreno J, Hartson L, Navarro C, Gaxiola M, Selman M, Randall TD. Inducible bronchusassociated lymphoid tissue (iBALT) in patients with pulmonary complications of rheumatoid arthritis. J Clin Invest. 2006;116(12):3183-3194.

5. Ruddle NH. Lymphatic vessels and tertiary lymphoid organs. J Clin Invest. 2014;124(3):953-959.

6. Mackay CR, Kimpton WG, Brandon MR, Cahill RN. Lymphocyte subsets show marked differences in their distribution between blood and the afferent and efferent lymph of peripheral lymph nodes. J Exp Med.1988;167(6):1755-1765.

7. Otsuki Y, Ito Y, Magari S. Lymphocyte subpopulations in high endothelial venules and lymphatic capillaries of bronchus-associated lymphoid tissue (BALT) in the rat. Am JAnat. 1989;184(2):139-146.

8. Hsiao HM, Li W, Gelman AE, Krupnick AS, Kreisel D. The role of lymphoid neogenesis in allografts. Am J Transplant. 2016;16(4):1079-1085.

9. Baddoura FK, Nasr IW, Wrobel B, Li Q, Ruddle $\mathrm{NH}$, Lakkis FG. Lymphoid neogenesis in murine cardiac allografts undergoing chronic rejection.
Am J Transplant. 2005;5(3):510-516.

10. Li W, et al. Lung transplant acceptance is facilitated by early events in the graft and is associated with lymphoid neogenesis. Mucosal Immunol. 2012;5(5):544-554.

11. Nasr IW, et al. Tertiary lymphoid tissues generate effector and memory T cells that lead to allograft rejection. Am J Transplant. 2007;7(5):1071-1079.

12. Li W, et al. Bronchus-associated lymphoid tissue-resident Foxp $3^{+} \mathrm{T}$ lymphocytes prevent antibody-mediated lung rejection. J Clin Invest. 2019;129(2):556-568.

13. Laidlaw BJ, et al. CD4 ${ }^{+} \mathrm{T}$ cell help guides formation of $\mathrm{CD} 13^{+}$lung-resident memory $\mathrm{CD}^{+} \mathrm{T}$ cells during influenza viral infection. Immunity. 2014;41(4):633-645.

14. Tanaka S, et al. IL-22 is required for the induction of bronchus-associated lymphoid tissue in tolerant lung allografts. Am J Transplant. 2020;20(5):1251-1261.

15. Reed HO, Wang L, Kahn ML, Hancock WW. Donor-host lymphatic anastomosis after murine lung transplantation. Transplantation. 2020;104(3):511-515.

16. Ruggiero R, et al. Reestablishment of lymphatic drainage after canine lung transplantation. J Thorac Cardiovasc Surg. 1993;106(1):167-171.

17. Joukov V, et al. A recombinant mutant vascular endothelial growth factor-C that has lost vascular endothelial growth factor receptor-2 binding, activation, and vascular permeability activities. J Biol Chem. 1998;273(12):6599-6602.

18. Huggenberger R, Ullmann S, Proulx ST, Pytowski B, Alitalo K, Detmar M. Stimulation of lymphangiogenesis via VEGFR-3 inhibits chronic skin inflam- mation. JExp Med. 2010;207(10):2255-2269.

19. Cui Y, et al. Therapeutic lymphangiogenesis ameliorates established acute lung allograft rejection. J Clin Invest. 2015;125(11):4255-4268.

20. Witmer AN, et al. VEGFR-3 in adult angiogenesis. J Pathol. 2001;195(4):490-497.

21. Saaristo A, et al. Lymphangiogenic gene therapy with minimal blood vascular side effects. J Exp Med. 2002;196(6):719-730.

22. Martel C, et al. Lymphatic vasculature mediates macrophage reverse cholesterol transport in mice. JClin Invest. 2013;123(4):1571-1579.

23. Pytowski B, et al. Complete and specific inhibition of adult lymphatic regeneration by a novel VEGFR-3 neutralizing antibody. J Natl Cancer Inst. 2005;97(1):14-21.

24. Thaunat $\mathrm{O}$, et al. Lymphoid neogenesis in chronic rejection: evidence for a local humoral alloimmune response. Proc Natl Acad Sci U S A. 2005;102(41):14723-14728.

25. Dashkevich A, et al. Lymph angiogenesis after lung transplantation and relation to acute organ rejection in humans. Ann Thorac Surg. 2010;90(2):406-411.

26. Kerjaschki D, et al. Lymphatic neoangiogenesis in human kidney transplants is associated with immunologically active lymphocytic infiltrates. JAm Soc Nephrol. 2004;15(3):603-612.

27. Källskog O, et al. Lymphatic vessels in pancreatic islets implanted under the renal capsule of rats. Am J Transplant. 2006;6(4):680-686.

28. Nykänen AI, et al. Targeting lymphatic vessel activation and CCL21 production by vascular endothelial growth factor receptor-3 inhibition has novel immunomodulatory and antiarterio- 
sclerotic effects in cardiac allografts. Circulation. 2010;121(12):1413-1422.

29. Dietrich T, et al. Cutting edge: lymphatic vessels, not blood vessels, primarily mediate immune rejections after transplantation. JImmunol. 2010;184(2):535-539.

30. Yin N, Zhang N, Xu J, Shi Q, Ding Y, Bromberg JS. Targeting lymphangiogenesis after islet transplantation prolongs islet allograft survival. Transplantation. 2011;92(1):25-30.

31. Krebs R, et al. Critical role of VEGF-C/VEGFR3 signaling in innate and adaptive immune responses in experimental obliterative bronchiolitis. Am J Pathol. 2012;181(5):1607-1620.

32. Gelman AE, et al. Cutting edge: acute lung allograft rejection is independent of secondary lymphoid organs. J Immunol. 2009;182(7):3969-3973.

33. Lakkis FG, Arakelov A, Konieczny BT, Inoue Y. Immunologic 'ignorance' of vascularized organ transplants in the absence of secondary lymphoid tissue. Nat Med. 2000;6(6):686-688.

34. Yamagami S, Dana MR, Tsuru T. Draining lymph nodes play an essential role in alloimmunity generated in response to high-risk corneal transplantation. Cornea. 2002;21(4):405-409.

35. Wang $L$, et al. Permanent survival of fully MHC-mismatched islet allografts by targeting a single chemokine receptor pathway. J Immunol. 2005;175(10):6311-6318.

36. Todd JL, et al. Hyaluronan contributes to bronchiolitis obliterans syndrome and stimulates lung allograft rejection through activation of innate immunity. Am J Respir Crit Care Med. 2014;189(5):556-566.

37. Huggenberger R, et al. An important role of lymphatic vessel activation in limiting acute inflammation. Blood. 2011;117(17):4667-4678.

38. Pedersen MS, et al. Lymphangiogenesis in a mouse model of renal transplant rejection extends life span of the recipients. Kidney Int. 2020;97(1):89-94.

39. Card CM, Yu SS, Swartz MA. Emerging roles of lymphatic endothelium in regulating adaptive immunity. JClin Invest. 2014;124(3):943-952.

40. Reed HO, et al. Lymphatic impairment leads to pulmonary tertiary lymphoid organ formation and alveolar damage. JClin Invest. 2019;129(6):2514-2526.

41. Karpanen T, et al. Lymphangiogenic growth factor responsiveness is modulated by postnatal lymphatic vessel maturation. Am J Pathol. 2006;169(2):708-718.

42. Shan J, et al. Do CD $4^{+}$Foxp $^{+}$Treg cells correlate with transplant outcomes: a systematic review on recipients of solid organ transplantation. Cell Immunol. 2011;270(1):5-12.

43. Brown K, Sacks SH, Wong W. Tertiary lymphoid organs in renal allografts can be associated with donor-specific tolerance rather than rejection. Eur J Immunol. 2011;41(1):89-96.

44. Graca L, Cobbold SP, Waldmann H. Identification of regulatory $\mathrm{T}$ cells in tolerated allografts. J Exp Med. 2002;195(12):1641-1646.

45. Barker CF, Billingham RE. The role of afferent lymphatics in the rejection of skin homografts. JExp Med.1968;128(1):197-221.

46. Hamano K, Rawsthorne MA, Bushell AR, Morris PJ, Wood KJ. Evidence that the continued presence of the organ graft and not peripheral donor microchimerism is essential for maintenance of tolerance to alloantigen in vivo in anti-CD4 treated recipients. Transplantation. 1996;62(6):856-860.

47. Okumi M, et al. Role of persistence of antigen and indirect recognition in the maintenance of tolerance to renal allografts. Transplantation. 2008;85(2):270-280.

48. Gershon RK, Kondo K. Infectious immunological tolerance. Immunology. 1971;21(6):903-914.

49. Kendal AR, et al. Sustained suppression by Foxp $3^{+}$regulatory $\mathrm{T}$ cells is vital for infectious transplantation tolerance.J Exp Med. 2011;208(10):2043-2053.

50. Muniz LR, Pacer ME, Lira SA, Furtado GC. A critical role for dendritic cells in the formation of lymphatic vessels within tertiary lymphoid structures. JImmunol. 2011;187(2):828-834.

51. Ran S, Montgomery KE. Macrophage-mediated lymphangiogenesis: the emerging role of macrophages as lymphatic endothelial progenitors. Cancers (Basel). 2012;4(3):618-657.

52. Chauhan SK, et al. A novel pro-lymphangiogenic function for Th17/IL-17. Blood. 2011;118(17):4630-4634.

53. Skobe M, et al. Induction of tumor lymphangiogenesis by VEGF-C promotes breast cancer metastasis. Nat Med. 2001;7(2):192-198.

54. Rangel-Moreno J, et al. The development of inducible bronchus-associated lymphoid tissue depends on IL-17. Nat Immunol. 2011;12(7):639-646.

55. Gräbner R, et al. Lymphotoxin beta receptor signaling promotes tertiary lymphoid organogenesis in the aorta adventitia of aged $\mathrm{ApoE}^{-/-}$mice. J Exp Med. 2009;206(1):233-248.

56 . Barone F, et al. IL-22 regulates lymphoid chemokine production and assembly of tertiary lymphoid organs. Proc Natl Acad Sci US A. 2015;112(35):11024-11029.

57. Joshi NS, et al. Regulatory T cells in tumorassociated tertiary lymphoid structures suppress anti-tumor T cell responses. Immunity. 2015;43(3):579-590.

58. Lee HJ, et al. Tertiary lymphoid structures: prognostic significance and relationship with tumour-infiltrating lymphocytes in triple-negative breast cancer. J Clin Pathol. 2016;69(5):422-430.

59. Okazaki M, et al. A mouse model of orthotopic vascularized aerated lung transplantation. Am J Transplant. 2007;7(6):1672-1679.

60. Li W, et al. Intravital 2-photon imaging of leukocyte trafficking in beating heart. J Clin Invest. 2012;(7):2499-2508.

61. Li W, et al. Orthotopic vascularized right lung transplantation in the mouse. J Thorac Cardiovasc Surg. 2010;139(6):1637-1643.

62. Singer T, et al. Left-sided mouse intubation: description and evaluation. Exp Lung Res. 2010;36(1):25-30.

63. Kreisel D, et al. In vivo two-photon imaging reveals monocyte-dependent neutrophil extravasation during pulmonary inflammation. Proc Natl Acad Sci U S A. 2010;107(42):18073-18078. 